\title{
THE STABILIZATION OF BUDKER-CHIRIKOV INSTABILITY BY THE SPREAD OF LONGITUDINAL VELOCITIES
}

\author{
Yu.Ya. Golub, \\ Moscow Radiotechnical Institute, 132 Warshavskoye shosse, 113519 Moscow, Russia, \\ E-mail: yurigolub@mtu-net.ru
}

\begin{abstract}
The two-beams electron - ion system consists of a nonrelativistic ion beam propagating co-axially with a high-current relativistic electron beam in a longitudinal homogeneous magnetic field [1]. The effect of spread of longitudinal velocities of an electron beam on instability Budker-Chirikov (BCI) in the system is investigated by the method of a numerical simulation in terms of the kinetic description of both beams. The investigations are development of investigations in [2].

Is shown, that the increasing of spread of longitudinal velocities of electron beam results in the decreasing of an increment of instability Budker-Chirikov and the increasing of length of propagation of a electron beam.
\end{abstract}

\section{BASIC EQUATIONS}

We investigate a two-beam electron-ion system consisting of a nonrelativistic ion beam propagating coaxially with a high-current relativistic electron beam. The both beams are injected in equilibrium into drift tube. The spread of longitudinal velocities of an electron beam are took place. The kinetic description- of both beams is provided by means of solutions of the Vlasov equations for the electron and ion distributions functions, $f_{\mathrm{e}, \mathrm{i}}(\mathrm{t}, \mathrm{z}, \mathrm{r}$, $\left.\mathrm{v}_{\mathrm{z}}, \mathrm{v}_{\mathrm{r}}, \mathrm{v}_{\theta}\right)$. The equations for the scalar potential and the three component of the vector potential are used for finding the electromagnetic fields. The equations are solved in the long-wave $\left(\partial^{2} / \partial \mathrm{z}^{2}<<\Delta_{\perp}\right)$, low-frequency $\left(\partial^{2} / \partial \mathrm{t}^{2}<<\mathrm{c}^{2} \Delta_{\perp}\right)$, axial-symmetric $(\partial / \partial \theta \equiv 0)$ case. where $\Delta_{\perp}$ is the transverse part of the Laplace operator. Boundary conditions for the potentials fellow from the system's axial symmetry, the presence of conducting tube with radius $\mathrm{R}$ and the gauge condition $\operatorname{div} \boldsymbol{A}=0$. The Vlasov equations are solved by the macroparticle method. It is assumed that the steady-slate process is periodic in time set with a frequency $\omega$. In this case it is convenient to use the longitudinal coordinate $\mathrm{z}$ as the independent variable, using the relation $\mathrm{d} / \mathrm{dt}=\left(\mathrm{l} / \mathrm{v}_{\mathrm{z}}\right) \mathrm{d} / \mathrm{dz}$, where $\mathrm{v}_{\mathrm{z}}$, is the velocity of a given macroparticle. The problem is then reduced to the evolution of a periodic-in-time system on $\mathrm{z}$.

The periodic in time (with frequency w) potential function are of the form

$$
G(t, z, r)=\bar{G}(z, r)+\operatorname{Re}\left\{\sum_{j} \tilde{G}(z, r) \cdot e^{i j \omega t}\right\}
$$

which is substituted into the equations for the potential components and integrated over a time period. The equations for the four components of the 4-poternial $A_{i}$, ( $\Delta_{\mathrm{ri}} A_{i}=4 \pi \rho_{\mathrm{i}}, \mathrm{i}=1,2,3,4 \Delta_{\mathrm{ri}}$ is the radial parts of the Dalamber operator, and $\rho_{\mathrm{i}}$ are the components of the 4density) are solved at every z--cross-section by the grid method.

The electron beam was divided into a number of fractions, which have different longitudinal velocities. The amount of fraction is odd number. Each fraction distinguish from another on the same velocity. One from the fractions have velocity, which is equal to the average electron beam velocity. The others fractions are symmetrical arrangement in pairs around of the fraction with average velocity. The quantity of the spread of longitudinal velocities of an electron beam is equal to difference between the last fraction and average velocity divided on average velocity and multiply on $100 \%$.

\section{BCI INSTABILITY}

The important of the instabilities in the two-beam electron-ion system are the Budker-may be solved by using of ion beam undulator. We investigate the ion beam undulator which Chirikov instability (BCI) $[3,4]$. They are connected with the resonance of the slowcyclotron wave of tile electron beam and the fast Langmure wave and fast betatron wave of the ion beam, respectively. Unlike [1], the spread of longitudinal velocities of an electron beam are took place.

The Budker-Chirikov instability take place in that case. The instability in the time periodic regime is displayed in the growth of the radial modulation amplitude of both beams along the longitudinal coordinate. Also the BCI is developed in exponential growth of the potential amplitude. The BCI increment decreases with the increase in spread of longitudinal velocities of an electron beam.

The dependence of space increment $\mathrm{E}$ of $\mathrm{BCI}$ on the spread of longitudinal velocities of an electron beam is shown in Fig. 1. The electron beam current $\mathrm{J}=0.5 \mathrm{kA}$, ion longitudinal velocity $b=0.03$, average relativistic factor $\mathrm{g}=3$, longitudinal magnetic field $1 \mathrm{kG}$, the drift tube radius $\mathrm{R}=1.5 \mathrm{~cm}$, ion beam density $n i=\mathrm{f}^{*} \mathrm{ne}, \mathrm{f}=1 / 9$, the electron and ion beam radii $=0.6^{*} \mathrm{R}$. The resonance frequency is to the increment maximum. 


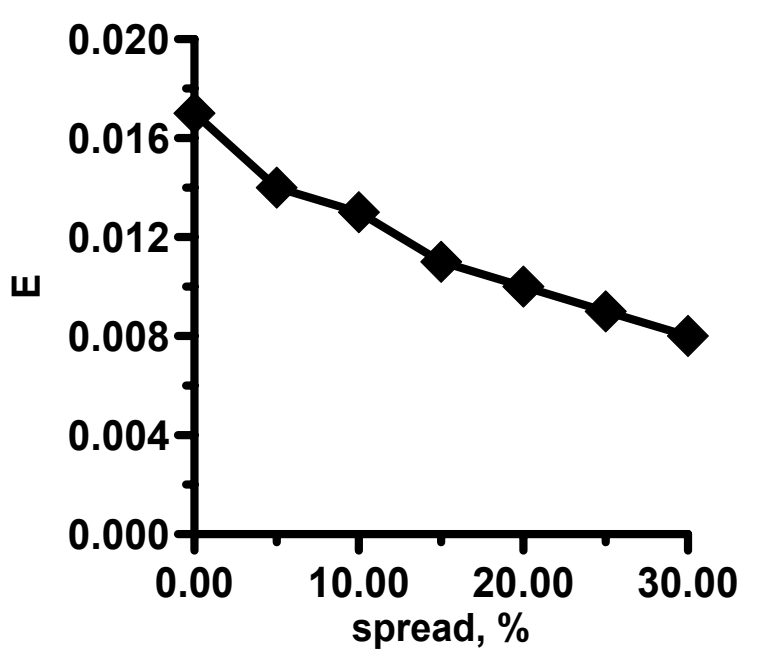

Fig.1: The dependence of space increment E of BCI on the spread of longitudinal velocities of an electron beam is shown in Fig. 1.

We see in Fig.1, that the BCI increment is decreased with decreasing of the ion beam radius. Because amount of electrons in resonance is increased.
If the spread of longitudinal velocities of an electron beam are large than $15 \%, \mathrm{BCI}$ is saturation by nonlinear mechanism [1]. Electron beam don't destroy, if the spread of longitudinal velocities of an electron beam are large than 15\%. Thus electron beam with the spread of longitudinal velocities must propagate without destruction with the ion beam.

\section{REFERENCES}

[1]. Yu.Ya. Golub, N.E.Rozanov, Nuclear Instruments and Methods in Physics Research, A358 (1995) 479

[2] Yu.Ya. Golub, Proceedings of EPAC 2002, Paris, France, 1497

[3] G.I. Budker. Atomnaya energiya 1 (1956) 9

[4] B.V. Chirikov, Atomnaya energiya 19 (1965) 239 